THOMAS SIMON

\title{
Gemeinwohltopik in der mittelalterlichen und frühneuzeitlichen Politiktheorie
}

\section{Zur Tradition der Politikliteratur}

Im Jahre 1533 erscheint in Marburg eine Schrift, die ausweislich ihres Titels ,Von dem Gemeinen Nutze" das politische Problem des Gemeinwohls zum Gegenstand einer eingehenden wissenschaftlichen Erörterung macht. Autor ist der Marburger Rechtsprofessor und mehrmalige Rektor der dortigen Juristenfakultät, Johannes Eisermann, alias Ferrarius, wie die im humanistisch beflissenen Stil dieser Zeit latinisierte Form des Namens lautet, unter dem der Autor bekannt geworden ist. ${ }^{1}$ Ferrarius wendet sich mit seinem Traktat an die ,Regenten und Untertanen“ aller „Communen“ und „Gemeinden"; so kann man es dem Untertitel der Schrift entnehmen. Sie ist dem Schwager des Autors gewidmet, einem Mann, der damals als Bürgermeister der zwischen Marburg und Frankenberg gelegenen Kleinstadt Wetter amtierte. Die städtischen Bürgermeister und Räte als Regenten ihrer Gemeinden sind der vorrangige Adressatenkreis, den Eisermann mit seinem Traktat ansprechen möchte. Der Autor schreibt also weniger für ein gelehrtes Publikum, sondern vorrangig für einen Personenkreis, „so zu regirn auffgeworffen“, und weil darunter ,die wenigsten latinisch kunnen oder in den freyen kunsten erfahren seyn", hat er seinen praxisorientierten Traktat nicht in der Gelehrtensprache, sondern in Deutsch verfaßt. ${ }^{2}$ Im Zuge seiner Abhandlung stößt Ferrarius schließlich auch zu einer ausdrücklichen Definition des „Gemeinen Nutz“ vor, wie er ihn verstanden wissen möchte und in seinem politischen Traktat verwendet: Im fünften Kapitel unter der Überschrift „Was der Gemein nutz sei“ stellt er fest, „,daß Respublica oder gemein nutz nit anders ist, denn ein gemein gute ordenung einer statt oder einer andern commun “. Im weiteren Verlauf der Schrift lassen sich dann bei näherem Hinsehen drei nebeneinander verwendete, aber fließend ineinander übergehende Bedeutungsvarianten des „Gemeinen Nutz" erkennen: Zum einen, wie sich schon der eben zitierten Definition entnehmen läßt, die „Respublica“ selbst, die ,burgerliche“ oder „menschliche Ge-

\footnotetext{
' Zur Person vgl. Stolleis 1988, S. $86 f$.

2 Vgl. Ferrarius 1533, Vorrede am Ende.

3 Vgl. ebd. S. 19v; vgl. Schulze 1986, S. 598 f.
} 
sellschaft", wie Ferrarius dieses Wort übersetzt. ${ }^{4}$ Zum anderen ein bestimmter Ordnungszustand, in dem sich dieses Gemeinwesen befinden soll, die "gute Ordnung“, schließlich aber auch das, was Ferrarius „die Gemeinschaft des Nutzes“ nennt: Es sind die „gemeinen Dinge“, die „allen gemein sind“, die sozusagen das materielle Substrat der „,burgerlichen Gesellschaft“ darstellen, also deren Gemeindegüter und Allmenden. Der ganze Traktat kreist um die Frage, wie sich der intendierte Ordnungszustand des "Gemeinen Nutz" in der „Respublica“ instituieren läßt, welche charakterlichen Vorbedingungen bei den Regenten und Untertanen dafür gegeben sein müssen, wie die Regierung zu diesem Ende zu führen ist und welche Ordnungsstrukturen das Gemeinwesen dazu im einzelnen aufzuweisen hat.

Was der Jurist und Universitätsprofessor aus Marburg hier unter dem Titel des „Gemeinen Nutz" veröffentlicht hat, ist nur dieses Titels wegen ungewöhnlich; der Sache nach handelt es sich um eine Arbeit aus einer im 16. Jahrhundert durchaus verbreiteten politischen Literaturgattung, die in der Forschung zur Verwaltungsgeschichte oder der politischen Ideengeschichte häufig unter den Bezeichnungen „Regiments-“ oder „Policeytraktate" zusammengefaßt werden. ${ }^{5}$ Diese Spezies praktischer Regierungs- und Verwaltungslehre ist wiederum nur eine spezifisch frühneuzeitliche Ausprägung einer seit dem Hochmittelalter gepflegten theoretischen Politikliteratur, die im wesentlichen mit der Aristotelesrezeption des 13. Jahrhunderts einsetzt; die Wiederentdeckung, Übersetzung und wissenschaftliche Erschließung der aristotelischen Politik durch die Scholastik war ein entscheidender Faktor für die Entstehung dieser Art der politischen Reflexion. ${ }^{6}$ Sie tritt im Laufe des Mittelalters und der frühen Neuzeit in ganz unterschiedlichen Literaturgattungen zutage. Im Mittelalter waren es in erster Linie die Fürstenspiegel, in denen sich neben der fürstlichen Tugendlehre unter anderem auch das Thema der rechten Ordnung des Gemeinwesens und seine sachgerechte und kunstfertige Leitung behandelt findet. ${ }^{7} \mathrm{Zu}$ den bekanntesten Werken dieser normativen Politikanleitung zählt vor allem der Fürstenspiegel des Thomas von Aquin, der es zuerst unternommen hat, die Leitlinien der aristotelischen Politik für die mittelalterliche Staats- und Herrschaftslehre zu erschließen und mit der kirchlichen Tradition zu verbinden; das Ergebnis hat er in die traditionelle Form eines Fürstenspiegels unter dem üblichen Titel De regimine principum gegossen. ${ }^{8}$ Was die Verbreitung anbelangt, steht allerdings unangefochten der am Ende des 13. Jahrhunderts verfaßte Fürstenspiegel des Augustiner-Eremiten und französischen Prinzenerziehers Aegidius Romanus an der Spitze; diese Regierungslehre wurde in nahezu alle europäischen Sprachen übersetzt und gilt als die meistgelesene Politik des Mittelalters überhaupt. ${ }^{9}$

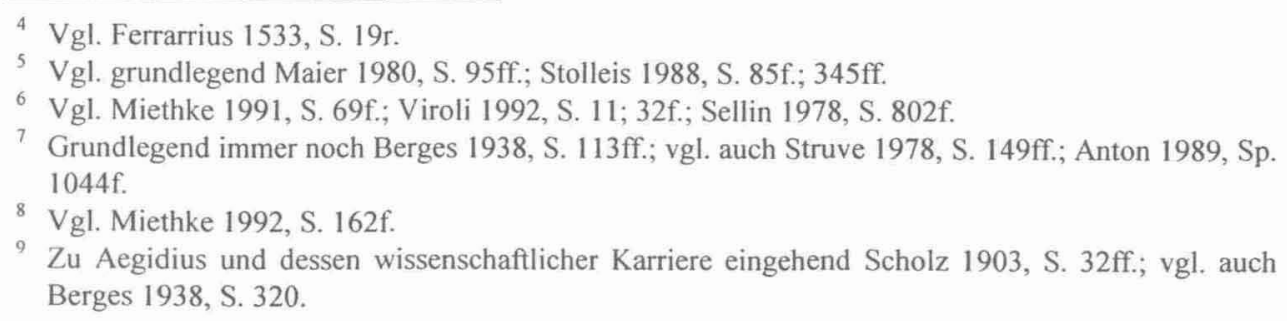


Neben die Fürstenspiegel treten dann zu Beginn der Neuzeit die Regimentstraktate, die sich - mit entsprechenden Inhalten - nicht mehr ausschließlich an den Princeps, sondern generell an alle Obrigkeiten einschließlich der städtischen Räte wenden und deren Aufgabenkataloge formulieren. ${ }^{10}$ Seit dem Ende des 16. Jahrhunderts entsteht dann schließlich eine auch akademisch-universitär verankerte Politikliteratur, die auf der Basis eines breiten Spektrums antiker Bezugstexte die Regierungskunst und deren Handlungsziele im wissenschaftlichen Stil der Zeit erörtert. ${ }^{11}$ Gemeinsam ist allen diesen literarischen Erscheinungsformen politischer Reflexion neben der Erörterung der Herrschaftsfunktionen vor allem auch das Thema des richtigen und daher von der Regierung anzustrebenden politischen Ordnungszustandes: Hier wird in allgemeiner Weise formuliert, welches die entscheidenden Kriterien der inneren Ordnung des Gemeinwesens sein sollen.

\section{II. bonum commune und die hochmittelalterliche Funktionserweiterung politischer Herrschaft}

Solcherlei Politikliteratur erweist sich auch als aussagekräftig hinsichtlich des Versuchs, die Bedeutung und den Stellenwert des Gemeinwohlargumentes im politischen Diskurs der Vormoderne zu bestimmen. In die Erörterung der Staatsfunktionen, der richtigen Verfassung des Gemeinwesens und der Kunst seiner Regierung ist das Problem des Gemeinwohls in mannigfacher Weise miteingeflochten. Typischerweise sind es dabei drei Wortgruppen, in denen sich der Gemeinwohlgedanke ausgedrückt findet: Die eine davon besteht aus begrifflichen Verbindungen mit dem Adjektiv „publica“, vor allem in der Kombination utilitas publica oder salus publica. Diese Wendungen finden sich schon im Römischen Recht und fließen von hier aus in die mittelalterlichen Rechtstexte und Urkunden. Eine zweite Wortgruppe zur Bezeichnung des Gemeinen Wohls ist um das Wort „bonum" gebildet, etwa bonum commune, bonum multitudinis oder bonum publicum. Solche Gemeinwohlformeln werden, soweit ersichtlich, vor allem in der wissenschaftlichen Politiklehre gebraucht, wie sie im Hochmittelalter auf der Grundlage der Aristotelesrezeption allmählich emporkommt. Mindestens ebenso wichtig wie die genannten rechts- und politikwissenschaftlichen Ausdrücke ist aber schließlich der volkssprachliche Ausdruck Gemeiner Nutz; er findet sich nicht nur in den deutschsprachigen Regimentstraktaten des 16. Jahrhunderts, wie sie von der eingangs dargestellten Schrift Eisermanns repräsentiert werden, sondern ebenso in zahllosen politischen Manifesten und Streitschriften dieser Zeit. ${ }^{12}$

Beobachtet man nun den Kontext und die Verwendungsweise dieser Formeln, so muß zunächst auffallen, daß dem Topos vom bonum commune erst in den scholastischen Fürstenspiegeln des hohen Mittelalters eine wesentliche Bedeutung zukommt. ${ }^{13}$ Natürlich taucht dieser Gemeinplatz auch im früheren Mittelalter vielfach dort auf, wo

\footnotetext{
${ }^{10}$ Vgl. dazu eingehend Simon 2002, Teil B, Kap. II.

11 Vgl. Stolleis 1988, S. 104ff.

12 Blickle 1986, S. 54lf.

13 Vgl. Hibst 1991, S. 174, 177 und 184 f.
} 
das Amt des Königs und seine Pflichten und Aufgaben beschrieben werden. Aber erst in den Fürstenspiegeln des 13. Jahrhunderts rückt das gemeinwohlorientierte politische Handeln zur primären, noch vor der Gerechtigkeit rangierenden Fürstentugend auf. In den älteren Quellen war es demgegenüber noch die justitia gewesen, die den Kanon der Fürstentugenden angeführt hatte. Umgekehrt definieren die scholastischen Fürstenspiegel den Tyrannen gerade mit der fehlenden Gemeinwohlbindung seines politischen Handelns: Der Fürst wird dann zum Tyrannen, wenn er statt des bonum commune sein bonum proprium sucht. ${ }^{14}$

Auffallenderweise taucht die Formel vorrangig in Verbindung mit einer ganz bestimmten Funktion des Fürsten auf - einer Funktion, die in den Fürstenspiegeln mit den Worten ,ordinare“, „,isponere“ oder auch „,dirigere“ umschrieben wird: Die Hauptaufgabe des Princeps besteht diesen Fürstenspiegeln zufolge darin, sein Land „ordinare in bonum commune", wie es etwa bei Aegidius Romanus heißt. ${ }^{15}$ Das "ordinare ${ }^{\text {" }}$ und seine begrifflichen Entsprechungen „disponere“ und „dirigere“ umschreiben eine damals neue Funktion weltlicher Herrschaft, die man als legislative Ordnungsfunktion bezeichnen könnte. ${ }^{16}$ In den älteren Funktions- und Aufgabenbeschreibungen wird man diese Form der Regierungstätigkeit noch vergeblich suchen - hier wird das herrschaftliche Aufgabenspektrum noch ganz von der Schutzleistung und der Rechtswahrung geprägt: ,justitiam expedire“, „corrigere" und „,defendere" sind die wesentlichen Begriffe, mit denen die Zwecke und Funktionen weltlicher Herrschaft hier umschrieben werden. Entscheidend ist, daß diese Funktionsformeln den Herrscher nirgendwo als Gesetzgeber ansprechen. ${ }^{17}$ Der Fürst soll ,legem servare“, soll dem Recht Geltung verschaffen, aber nicht in die Normenordnung eingreifen. Genau dies aber - die Normgebung - macht zu einem entscheidenden Teil die neuartige Ordnungsfunktion aus: Das entscheidende Handlungsinstrument ist dabei die Gesetzgebung - die scholastischen Fürstenspiegel sprechen hier von "ordinationes facere“" ${ }^{18}$ nicht von „, legem dare“. „Ordinationes face$r e^{\prime \prime}$ ist der Erlaß von Gesetzen zur Bewahrung der Guten Ordnung des Gemeinwesens; im Kern ist hier also genau diejenige herrschaftliche Tätigkeitsform angesprochen, die Jahrhunderte später in Bezug auf die deutschen Territorien mit dem Terminus der „Policey" verbunden wird. Ziel des ordinare ist die „politia bene disposita“, wie es Tolomäus von Lucca in seiner Fortsetzung des thomasianischen Fürstenspiegels ausdrückt. ${ }^{19}$ Sie soll mittels einer entsprechenden Gesetzgebung realisiert werden, mit der die malae consuetudines, welche die gute Ordnung stören, unter Strafandrohung verboten werden. Der Gedanke, daß man bestimmte, politisch erwünschte Ordnungszustände im Wege der Gesetzgebung bewahren, ja sogar neu konstituieren könne, wird in der mittelalterlichen Welt zuerst in der scholastischen Politikliteratur zum Gegenstand gelehrter Erörterung gemacht. ${ }^{20}$ Sie läuft zeitlich im wesentlichen parallel mit dem Beginn einer ent-

\footnotetext{
${ }^{14}$ Simon 2002, Teil A, Kap. III.3.

15 Aegidius Romanus 1556, S. 280

${ }^{16}$ Eingehend hierzu und zum folgenden Simon 2002, Teil A, Kap. III.3.

17 Vgl. Berges 1938, S. 14.

18 Aegidius Romanus 1556, 3. Buch, 2. Teil, Kap. 19.

19 Tolomäus 1948, IV, 23, S. 90b-91a.

${ }^{20}$ Grundlegend Gagnér 1960, S. $193 \mathrm{ff}$.
} 
sprechenden legislativen Praxis in den oberitalienischen Städten seit dem 13. Jahrhundert - einer Praxis, die von den großräumigeren Territorialstaaten in der Regel erst am Ende des Mittelalters aufgegriffen wird. ${ }^{21}$

Es fällt auf, daß die Intensivierung der Gemeinwohltopik, wie sie in den scholastischen Fürstenspiegeln beobachtet werden kann, zeitlich einher geht mit der erstmaligen Thematisierung der legislativen Steuerungsfunktion als einem neuen Aufgabenfeld des Fürsten. Erst zusammen mit dem Leitbild des fürstlichen Gesetzgebers wird der bonum commune-Topos als bindender Leitgesichtspunkt fürstlichen Handelns in den Fürstenspiegeln sichtbar. Der Gemeinwohltopos vom bonum commune und seine begrifflichen Entsprechungen sind jedenfalls im Moment ihrer intensivierten Verwendung in den scholastischen Fürstenspiegeln eng mit der Ordnungsfunktion verbunden; sie macht den wesentlichen Kontext aus, in den sich die Gemeinwohlformeln hier plaziert finden.

\section{Gemeinwohltopos und Genossenschaften}

Das Bild kann noch geschärft werden, wenn man auch außerhalb der hier vorrangig thematisierten Politiktheorie den semantischen Kristallisationspunkten nachspürt, in deren Umfeld der Gemeinwohltopos in besonders dichter Weise verwendet wird. Hierzu liegen einige detaillierte Einzeluntersuchungen vor, unter denen vor allem die Arbeiten von Peter Blickle und Winfried Eberhard zu nennen wären. Beide zeigen mit reichem Quellenmaterial, wie die Gemeinwohlformel und ihre zahlreichen lateinischen und deutschsprachigen Äquivalente vorrangig im genossenschaftlichen Sozialkreis städtischer und dörflicher Gemeinden die politische und rechtliche Rhetorik prägten. ${ }^{22}$ Blickle geht dabei sogar soweit, die ,Wertkategorie des gemeinen Nutzens“ ausschließlich im „System des Kommunalismus“ zu verorten. Herrschaftsverhältnisse hingegen rechtfertigten sich ihm zufolge regelmäßig nicht mit der Funktion, den Gemeinen Nutzen zu fördern, sondern ausschließlich mit ihrer Aufgabe der Rechtssicherung und Friedenswahrung. ${ }^{23}$ Auch wenn sich diese Schlußfolgerung als übermäßig zugespitzt erweist, weil dabei, wie Eberhard zutreffend einwendet, die ältere, auf das Königtum orientierte Tradition der Begriffsverwendung aus dem Blick gerät, ${ }^{24}$ so erweist sich doch - jedenfalls für das Mittelalter - die These einer ausgeprägten Affinität des Gemeinwohltopos zu den genossenschaftlich strukturierten Sozialbereichen als richtig. Denn wenn es auch im früheren Mittelalter eine auf das antike Rechts- und Staatsschrifttum gestützte kontinuierliche Praxis der Gemeinwohlargumentation in den Urkundenformulierungen der fürstlichen Kanzleien gegeben haben mag, so ist die sprunghafte Intensivierung des Gemeinwohlrekurses während des Hochmittelalters ebenso unbestreitbar wie der Umstand, daß diese Intensivierung zunächst vorrangig im städtisch-kommunalen Raum stattfand. ${ }^{25}$ Und hier wiederum war es, wie gerade die zahlrei-

\footnotetext{
$21 \mathrm{Vgl}$. Simon 1997, S. $1204 \mathrm{f}$.

22 Vgl. Eberhard 1988, S. $271 \mathrm{f}$.

${ }^{23}$ Blickle 1986 S. $543 f$.

${ }^{24}$ Vgl. Eberhard 1985, S. 198ff. sowie 1986, S. 245f. und 1988, S. $273 \mathrm{f}$.

25 Vgl. hierzu auch Le Goff 1965, S. 204.
} 
chen, von Eberhard und Blickle referierten Quellenstellen eindringlich zeigen, vorrangig der Sachbereich der Normgebung, der immer wieder Anlaß zum legitimierenden Rückgriff auf die Kategorie des Gemeinwohls gab: Es war dasjenige politische Handeln, welches das Wiener Stadtrecht 1221 mit den Worten ,disponere de universis, quae ad utilitatem civitatis pertinent" ${ }^{26}$ umschreibt, also die Ordnung des städtischen Gemeinwesens durch Erlaß von Gassen-, Zunft-, Markt- oder gar umfassenden, in den lateinischen Quellen als statuta bezeichneten Stadtordnungen. Ihr Erlaß wurde in den Proömien regelmäßig unter Verweis auf das Gemeinwohl gerechtfertigt, das damit erhalten und gefördert werden sollte. ${ }^{27}$ Kommunale Bewegung und die Intensivierung der Gesetzgebungstätigkeit standen in engster Wechselwirkung miteinander und bedingten beide den steigenden argumentativen Stellenwert des Gemeinwohltopos, wie er für den Verlauf des Hochmittelalters zu beobachten ist. Vieles deutet darauf hin, daß eine entscheidende gesetzgebungsgeschichtliche Wurzel der policeylichen Ordnungstätigkeit in den genossenschaftlichen Sozialstrukturen zu suchen ist - jedenfalls außerhalb der Kirche, die auch in diesem Punkt eine weiter zurückreichende Tradition aufweist. Im strukturell verdichteten und ökonomisch am stärksten differenzierten Raum der Städte erwuchs am frühesten der Bedarf für eine neuartige Steuerungsgesetzgebung, die in spezifischer Weise darauf ausgerichtet war, Mißstände zu beseitigen, um dem Gemeinwesen auf diese Weise bestimmte Ordnungsstrukturen zu verleihen. Soziale Verdichtung und ausgeprägte funktionale Differenzierung bedingten hier einen vergleichsweise hohen Steuerungsbedarf, der mit dem Erlaß der Statuten und „Ordnungen“ befriedigt werden sollte. ${ }^{28}$ Aber auch der Sozialraum des Dorfes stellte sich auf Grund der - maßgeblich durch Dreifelderwirtschaft und Flurzwang bedingten - überaus engen ökonomischen Verknüpfung der einzelnen Bauernstellen und deren gemeinsamer Einordnung in den kräftig ausgeprägten Kollektivwirtschaftsrahmen der Allmenden als Sozialgebilde mit hohem internem Regelungsbedarf dar, der sich in den genossenschaftlichen Vereinbarungen der "Einungen" oder „Willküren" niederschlug. ${ }^{29}$ Diese gemeindeinterne Regulierungstätigkeit läßt sich der Sache nach seit dem Hochmittelalter belegen, und nicht zufällig wurde gerade in diesem Punkt neben dem städtischen Sozialkreis ein weiteres Feld auffallend vielfältiger und häufiger Verwendung der Legitimationsformel vom Gemeinen Nutzen gefunden. ${ }^{30}$ Den genossenschaftlichen Verbänden städtischer und ländlicher Provenienz war aber nicht nur ein besonders hoher Regelungsbedarf eigen, sondern sie waren auch in besonderer Weise zur normativen Regulierung befähigt, weil das Konsensprinzip bei ihnen ein wesentliches Strukturelement ausmachte, das gerade auch bei der Normproduktion zur Geltung kam. Eingespielte Konsensmechanismen waren aber im Mittelalter eine ganz wesentliche Vorbedingung für die Statuierung "neuer" Normen, insbesondere dann, wenn sie zum überlieferten Recht in Widerspruch traten. Das änderte sich im Grundsatz auch dann nicht, als am Ende des Mittelalters die nunmehr erstarkenden Territorialherrschaften einen Teil der legislativen Steuerungstä-

\footnotetext{
${ }^{26}$ Zit. nach Eberhard 1988, S. 280.

27 Zahlreiche anschauliche Einzelbeispiele bei Eberhard 1988, S. $280 \mathrm{ff}$.

28 Simon 1997, S. 1209ff.

29 Vgl. dazu Vogel 1953, S. $52 \mathrm{ff}$.

30 Bader 1962, S. 334ff.; Blickle 1986, S. 542.
} 
tigkeit an sich zogen und eine Regulierung nunmehr in territorialem, d.h. in ,policeylichem" Rahmen versuchten: Es ist längst offenbar, daß auch dies, jedenfalls noch im 16. Jahrhundert, keine nur obrigkeitliche, rein befehlsgestützte, sondern eine gleichfalls auf das stärkste von konsensualen Mechanismen getragene Angelegenheit war. ${ }^{31}$ Um so mehr waren im Mittelalter die verschiedenen genossenschaftlich strukturierten Verbände, angefangen bei den Städten mit ihren vielfältigen, wiederum genossenschaftlich strukturierten Untergliederungen, bis hin zu den zahllosen Dorf- und Nutzungsgenossenschaften zur normativen Steuerung prädestiniert, weil sie der konsensualen Willensbildung einen institutionellen Rahmen geben konnten und damit zugleich auch eine unerläßliche Vorbedingung für die Implementierung der neustatuierten Steuerungsnormen schufen. Eben weil es sich bei den genossenschaftlich strukturierten Sozialbereichen um gleichermaßen regelungsintensive wie implementationsfähige soziale Einheiten handelte, kam hier dem Gemeinwohlargument auch jener übereinstimmend beobachtete überragende Stellenwert zu. Wie in der eingangs behandelten Politikliteratur steht der Gemeinwohltopos auch hier, im Kontext seiner genossenschaftlichgemeindlichen Verwendung, in engem Bezug zur Mobilisierung der Normenordnung im Wege der Gesetzgebung, die hier wie dort unter Verweis auf den Gemeinwohlgedanken legitimiert wird. Die großen Fürstenspiegel des 13. Jahrhunderts hatten Gesetzgebung und Gemeinwohl zwar als politische Aufgabe des Fürsten, also im Kontext monarchischer Herrschaft thematisiert, aber es spricht sehr viel dafür, die Gesetzgebungslehre, wie man sie etwa bei Thomas oder Aegidius ausformuliert findet, in engem Zusammenhang mit der zeitgenössischen Statutenpraxis der oberitalienischen Städte zu sehen. ${ }^{32}$ Es ist daher fraglich, inwieweit wirklich von einer „Usurpation" des Gemeinwohlbegriffs durch Städte und Landstände während des Spätmittelalters oder einem „Eingreifen genossenschaftlicher Instanzen in die Staatszweckbestimmung des gemeinen Nutzens " gesprochen werden kann. ${ }^{33}$ Denn in solchen Aussagen ist die Vorstellung enthalten, es habe ursprünglich eine Art fürstliches Definitionsmonopol gegeben, auf Grund dessen die Fürsten das Gemeinwohl für ihre Länder jeweils einheitlich bestimmen konnten. Dies setzt aber ein Maß an Zentralisierung und eine Fähigkeit zur Normsetzung und -durchsetzung voraus, die auf der territorialstaatlichen Ebene im Laufe des späteren Mittelalters erst ganz allmählich heranwuchsen. Treffender dürfte es sein, für diesen Zeitraum von einem allmählichen Emporsteigen der Regulierungstätigkeit von der lokalen, gemeindlich-genossenschaftlichen Ebene auf die sich langsam verdichtende staatliche Ebene auszugehen ${ }^{34}$ - ein Vorgang, der in der Verortung des Gemeinwohlrekurses seine Parallele findet: Neben der Gemeinwohlargumentation der „Kronjuristen $^{635}$ stand die aus den eigenständigen Regelungsbedürfnissen der Kommunen erwachsende Topik vom Gemeinen Nutzen. Erst mit dem Erstarken des Territorialstaates und seiner Regelungstätigkeit der „Policey“ gegen Ende des Mittelalters kam es über der Frage zum Konflikt, wem die Konkretisierung und Bestimmung des Gemeinwohls

31 Vgl. Mohnhaupt 1972, S. 191; Blickle 1986, S. 541.

${ }^{32}$ Vgl. Simon 2002, Teil I, Kap. III.5.a.

33 Eberhard 1985, S. 203 sowie 248.

${ }^{34} \mathrm{Vgl}$. Simon 1997, S. 1205 f.

35 Jakobs 1994, S. 10. 
im Wege der Gesetzgebung zukomme - Fürst und „Staat“ oder den verschiedenartigen genossenschaftlichen Zusammenschlüssen? Die These von Blickle, in der Adelsgesellschaft des Mittelalters gebe es „den Gemeinnutz als anerkannten gesellschaftlichen Wert nicht ausdrücklich" ${ }^{36}$ erweist sich damit im Kern als zutreffend: Die traditionellen Funktionen adeliger Herrschaftsübung, der Schutz und die Rechtswahrung, mußten augenscheinlich nicht in gleichem Maße unter Rückgriff auf das Gemeinwohlargument legitimiert werden, wie die Normgebung.

Außerhalb der Städte war es im Mittelalter vor allem die Landfriedensgesetzgebung, die einen auffallenden Bezug zum Gemeinwohlargument aufwies. Diese noch ganz durch konsensuale Mechanismen untermauerte Form der Gesetzgebung ${ }^{37}$ kann man als territoriales, auf den ländlichen Raum bezogenes Seitenstück der städtischen Steuerungsgesetzgebung auffassen: Im großräumigen Rahmen der Territorien konnte das politische Instrument der Normgebung vorerst nur auf einzelnen Regelungsfeldern eingesetzt werden, unter denen die Sicherung des Landfriedens durch räumliche und örtliche Eindämmung der Fehdeübung einen bevorzugten Platz einnahm. Auch in diesem Kontext wurde die Gesetzgebung entscheidend mit dem Gemeinwohlargument legitimiert. $^{38}$

\section{Die Bedeutung des Gemeinwohltopos im Kontext des mittelalterlichen Gesetzgebungsdiskurses}

Der Gemeinwohltopos weist also, soweit man seine Verwendung in der frühen Politikliteratur des hohen Mittelalter ins Auge faßt, eine besondere Affinität zum neuartigen politischen Handlungsfeld gesetzgebender und normschöpfender Tätigkeit auf. Beobachtet man die intentionale Stoßrichtung des Gemeinwohlargumentes in diesem Kontext genauer, so lassen sich nebeneinander zwei Funktionen erkennen:

Es fungierte zunächst als eine Richtungsvorgabe, die den Kurs der legislativen Steuerung bestimmen und die normgebenden Instanzen zugleich darauf festlegen sollte. In den Gemeinwohlformeln waren demgemäß die jeweiligen politischen Wertvorstellungen von der richtigen gesellschaftlich-sozialen Ordnung miteingeschlossen - hierin überschnitten sie sich inhaltlich mit der Zielformel der politia bene disposita, die in der frühneuzeitlichen "Guten Policey" weiterlebte. Es ist diese Bedeutungsvariante angesprochen, wenn ein Autor wie etwa Ferrarius den „Gemeinen Nutz" mit der „Guten Ordnung einer Stadt oder eines anderen Gemeinwesens" gleichsetzt. Die „gute Ordnung", die hier anvisiert wird, bestand im Kern aus einer Summe von überlieferten Pflichten- und Funktionszuweisungen, die jedem Stand und Beruf einen bestimmten Ort im gesellschaftlichen Gefüge gab. „Gut" war die Ordnung dann, wenn sich alle Gesellschaftsglieder innerhalb ihrer jeweils vorbestimmten Pflichten- und Funktionsbereiche hielten, wenn jedermann, so drückt es Ferrarius aus, ,auff sein ampt und beruffung

36 Blickle 1986, S. 543.

37 Zur Bedeutung der Landfrieden: Kaufmann 1979 und Fried 1969.

38 Vgl. Gierke 1868, S. 505. 
sihet" und „keines dem andern in den beuelch greifft" ${ }^{39}$ Ein solches Ordnungsleitbild basiert auf der Vorstellung fester, grundsätzlich dem Menschen vorgegebener sozialer Strukturen, in die er sich einzufügen hat. Derartige Leitvorstellungen „guter Ordnung“" finden sich auch schon in den scholastischen Fürstenspiegeln des 13. Jahrhunderts. ${ }^{40}$ Dort ist es der zentrale Begriff der pax, in dem alle Ordnungsprojektionen zusammenlaufen: Der Gehalt der pax im mittelalterlichen Sinne erschöpfte sich nicht in der bloßen absentia belli, sondern umfaßte darüber hinaus die Ordnungsvorstellung der tranquillitas ordinis, die nach der klassischen, das ganze Mittelalter hindurch weitergegebenen Formel Augustins darin bestand, daß jeder Teil des Kosmos den ihm im Gesamtplan der Schöpfungsordnung zugedachten Platz einnimmt - eben dies ist die „parium dispariumque rerum sua cuique loca tribuens dispositio“".41 Das bonum commune, das der Fürst unter anderem auch mit dem Mittel der Gesetzgebung ansteuern sollte, bestand in der Realisierung und Durchsetzung dieser $p a x .^{42}$ In der Formel vom Gemeinwohl schwingen diese maßgebenden politischen Ordnungsvorstellungen mit. Sie hatte in diesem Zusammenhang zugleich einen ausgeprägt normativen Gehalt, indem sie die Träger der politischen Herrschaft - die städtischen Obrigkeiten gleichermaßen wie die Fürsten auf die Realisierung solcher Ordnungsvorstellungen festzulegen suchte.

Es liegt nahe, die auffallende Aktivierung des Gemeinwohlgedankens in der mittelalterlichen Politikliteratur als Reaktion auf eine Vergrößerung des politischen Handlungsspielraumes zu deuten. Denn mit der neuen Funktion des legislativen Ordnens wurden die traditionellen mittelalterlichen Herrschaftsfunktionen der Schutzleistung und der Rechts- und Friedenswahrung beträchtlich ausgeweitet. Darin lag zugleich auch eine Intensivierung der Herrschaft, weil die Normenordnung der Gesellschaft nunmehr gezielter politischer Einwirkung zugänglich geworden war: Durch die Normgebung wurde sie - jedenfalls in ersten Ansätzen - zum Produkt politischer Gestaltung. Die neuartige Steuerungsfunktion bewirkte eine verstärkte Einwirkung auf die Gesellschaft - eine Einwirkung, die in ihrer Intensität weit über dasjenige hinausging, was mit den überkommenen jurisdiktionellen und schutzgewährenden Herrschaftsfunktionen verbunden war und die sich eben deshalb auch am ehesten in kleinräumigen, auf dem Fundament konsensualer Mechanismen organisierten politischen Gemeinschaften realisieren ließ. Denn die traditionellen Aufgaben weltlicher Herrschaft im Mittelalter beinhalteten keine Regulierung der Gesellschaft, hatten also die gesellschaftliche Normenordnung unberührt gelassen und waren darauf beschränkt gewesen, diese, auch den Herrschaftsträgern im wesentlichen vorgegebene Normenordnung, im Rahmen ihrer richterlichen Funktion zu schützen und durchzusetzen. Nun aber wurde die Normenordnung selbst zum Gegenstand politischer Gestaltung, die viele gesellschaftliche Bereiche erfaßte und den gängigen Ordnungsvorstellungen gemäß zu beeinflussen versuchte. Dieser Vorgang scheint das Bestreben erzeugt zu haben, den sich öffnenden politischen Gestaltungsspielraum mit einem intensivierten Rückgriff auf die Gemeinwohltopik zu kompensieren. Die vergrößerte politische Dispositionsfreiheit weltlicher Herrschaft sollte dadurch

${ }^{39}$ Ferrarius 1533 , S. 61 und S. 9r.

${ }^{40}$ Hierzu eingehend Simon 2002, Teil A, Kap. II.

${ }^{41}$ Augustin 1981, Buch XIX, Kap. 13.

${ }^{42}$ Zum „, bonum commune des Friedens" siehe auch Schneider 1990, S. $55 \mathrm{ff}$. 
eingefangen und umfriedet werden. Die Funktion des Gemeinwohltopos ist hier vergleichbar mit derjenigen des Naturrechts, das ebenfalls darauf ausgerichtet war, legislative Entscheidungsspielräume zu begrenzen. Es ist wohl kaum als Zufall zu betrachten, daß gleichzeitig mit der Aufwertung der Gemeinwohlformeln auch die kanonistische und scholastische Naturrechtsdebatte einsetzte. ${ }^{43}$ Auch sie stellt sich als Widerhall beginnender legislativer Beeinflussung der Normenordnung dar, die durch Formulierung indisponibler Basisrechtssätze begrenzt und angeleitet werden sollte.

Das neuartige Handlungsinstrument der Gesetzgebung und die damit verbundene Intensivierung politischer Herrschaft bewirkte aber nicht nur das Bedürfnis nach deren normativer Bindung, sondern ebenso einen erhöhten Legitimationsbedarf. An vielerlei Punkten läßt sich erkennen, daß man die Gesetzgebung im Mittelalter als ein Ausnahmephänomen betrachtete. Wie skeptisch dieses politische Handlungsinstrument damals eingeschätzt wurde, erschließt sich aus der mittelalterlichen Gesetzgebungslehre, wie man sie etwa im Fürstenspiegel des Aegidius Romanus, breiter ausgeführt aber in der Summa theologica des Aquinaten findet: Obwohl beide Autoren der Gesetzgebung unter den fürstlichen Aufgaben einen hohen Rang einräumen, raten sie dennoch grundsätzlich von seinem Einsatz ab: Auch der scholastischen Aristoteles-Exegese, die gemeinhin als Vorreiter einer gesetzgebungsorientierten Politik gilt, merkt man deutlich an, daß die Normgebung als ein neuartiges Ordnungsinstrument betrachtet wurde, dessen Einsatz zu dem nach wie vor das Rechtsbewußtsein beherrschenden Grundsatz im Widerspruch stand, daß die Normenordnung möglichst unbeeinflußt zu lassen sei. ${ }^{44} \mathrm{Die}$ scholastische Rechts- und Politiktheorie suchte die neuartige Gesetzgebungstätigkeit mit dem überkommenen Rechtsdenken dadurch in Übereinstimmung zu bringen, daß sie zum einen die Fälle politisch empfehlenswerter Gesetzgebung auf ein Minimum reduzierte, diese aber zum anderen in den verbleibenden Ausnahmesituationen, in denen dem Fürst der Einsatz des legislativen Ordnungsinstrumentes empfohlen werden kann, mit dem Gemeinplatz vom bonum commune zu rechtfertigen suchte: Das Gemeinwohl, so Thomas in der Summa, erzwinge in Einzelfällen den Einsatz der Gesetzgebung, weil sich die tatsächlichen Verhältnisse der Gesellschaft ständig veränderten, was eine begrenzte Anpassung des Normenbestandes an die gewandelten Verhältnisse zur Wahrung des Gemeinen Wohls erforderlich machen könne. ${ }^{45}$

\section{Das Gemeinwohlargument im Zeitalter der Staatsräson}

Das Wechselspiel herrschaftsbindender wie -legitimierender Argumentation, wie sie die Verwendung des Gemeinwohltopos in den mittelalterlichen Fürstenspiegeln und den Regimentstraktaten zu Beginn der Neuzeit prägt, findet sich auch in der Politikliteratur des späteren 16. und 17. Jahrhunderts wieder. Allerdings ist hier nunmehr vielfach eine signifikante Akzentverschiebung festzustellen. Jedenfalls schiebt sich nun in Teilen der

\footnotetext{
${ }^{43} \mathrm{Vgl}$. Gagnér 1960, S. 185ff.

${ }^{44}$ Eingehend Simon 2002, Teil A, Kap. III.5.b.

45. Gagnér 1960, S. 275f., $279 f$.
} 
Literatur der Gesichtspunkt der Herrschaftslegitimierung in prägnanter Weise in den Vordergrund.

Die Staats- und Politiklehre erfuhr im Laufe des 16. Jahrhunderts einen tiefgehenden Umbruch, in dessen Verlauf die Ziele politischen Handelns in grundlegender Weise reformuliert wurden. ${ }^{46}$ Diese neue Form des politischen Diskurses nahm in Italien ihren Ausgang. ${ }^{47}$ Bis heute sieht man in den beiden Hauptwerken des Niccoló Machiavelli, den „Discorsi“ und dem in die äußere Form eines Fürstenspiegels gekleideten „Principe“, eine entscheidende Wurzel dieser neuen Form politischen Denkens. ${ }^{48}$ In Deutschland wurde es im wesentlichen erst seit dem 17. Jahrhundert rezipiert. Dabei muß man freilich sorgfältig differenzieren: Die deutsche Politiklehre rezipierte das neuartige politische Denken im Zuge eines komplexen Anverwandlungsvorganges, bei dem das Neue zu großen Teilen in das konventionelle ältere Politikkonzept eingefügt und dabei in Teilen so verändert wurde, daß es damit kompatibel erschien. ${ }^{49}$ Die deutschen Regimentstraktate bis zur Mitte des 16. Jahrhunderts, etwa eines Johannes Oldendorp, Johannes Ferrarius oder Georg Lauterbeks, zeigen sich von diesem Vorgang noch gänzlich unberührt; sie gingen im wesentlichen noch von den scholastischen Politikgrundsätzen aus, die sie - schon durch ihren Gebrauch der deutschen Sprache - für die Verwaltungspraxis der deutschen Territorien zugänglich und applikabel machten. ${ }^{50}$ Vor allem durch die gewaltige wissenschaftliche Autorität des auch in Deutschland lehrenden Niederländers Justus Lipsius wurde dann aber ausgangs des 16. Jahrhunderts die neue Politiklehre auch in Deutschland einflußreich. ${ }^{51}$ Als deren Herzstück darf die Lehre von der Staatsräson betrachtet werden. ${ }^{52}$ Sie ist - vor allem aus der deutschen Perspektive - untrennbar mit dem Namen Giovanni Boteros verbunden; er hat im Jahre 1586 als erster eine politische Handlungsanweisung unter dem Titel der „Staatsräson“ veröffentlicht. Vor allem durch dieses Werk wurde die Lehre von der ragione di stato auch nördlich der Alpen bekannt; schon am Ende des 16. Jahrhunderts lagen Übersetzungen dieses Buches vor.

Es kann hier nicht auf alle Aspekte dieser neuen Politiklehre eingegangen werden, denn nicht sie ist hier das Thema, sondern die Frage, inwieweit sich nun im Kontext der politiktheoretischen Umbewertungen, wie sie jedenfalls partiell seit dem Ende des 16. Jahrhunderts auch in Deutschland zu beobachten sind, die Verwendungsweise und der Gehalt der verschiedenen Gemeinwohltopoi veränderte. Unter diesem Gesichtspunkt betrachtet, ist es vor allem wesentlich, daß die Lehre von der Ratio status - jedenfalls in ihrem Ursprung - nicht mehr auf die respublica oder die politia als Ganzes, sondern auf den ,status" bezogen war. Und dieser ,status", dessen Eigeninteressen und dessen Gesetzmäßigkeiten seiner Stärkung die Staatsräson-Literatur in der Nachfolge Boteros erörterte, war - so wie ihn jedenfalls Botero aufgefaßt hat - etwas durchaus anderes als

\footnotetext{
46 Vgl. Sellin 1978, S. 810; Behnen 1987, S. 137.

${ }^{47}$ Viroli 1992, S. 136ff.

48 Literaturhinweise bei Stolleis 1981, S. 146f.

49 Stolleis 1980, S. 53 und 66; Stolleis 1983 a, S. 76; für Arnisäus: Dreitzel 1970, S. 398.

${ }^{50} \mathrm{Vgl}$. Simon 2002, Teil B, Kap. III.1 und 2.

51 Stolleis 1987, S. 243ff

52 Stolleis 1988, S. 197ff.; Sellin 1978, S. 809; Dreitzel 1995, S. 134f.
} 
die respublica oder die politia, die den Gegenstand der traditionellen Politiklehre abgab. ${ }^{53}$ Botero etwa umschreibt den ,status" mit dem Wort ,dominium ${ }^{c 6}:^{54}$ Der ,status" als das „dominium “ des Fürsten ist in diesem Kontext nichts anderes als dessen persönliche Herrschaft und Macht. Deren materieller Kern sind die Güter und das Vermögen des Fürsten; den Finanzen kam demzufolge im Kontext dieses Politikverständnisses ein ganz neuartiger Stellenwert zu: Nicht zufällig ist es die Staatsräson-Literatur, wo der Topos vom Geld als dem Nerv aller Dinge erstmalig auftaucht. ${ }^{55}$ Wesentlich ist, daß die von der Staatsräsonlehre ausgearbeiteten politischen Handlungsstrategien - anders als dies bei der zu Beginn besprochenen Politiktheorie der Fall war - nicht mehr die gute Ordnung des Gemeinwesens insgesamt, sondern vorrangig den status, also die Macht des Fürsten und deren Steigerung und Stärkung, im Auge haben; auch dies ließ sie vom Standpunkt eines traditionellen Politikverständnisses aus geradezu als Provokation erscheinen. Nicht mehr um die „politia bene disposita“ oder die „gute Policey“ geht es in dieser Form der Machtpolitik, sondern um das „,dominio fermo“, wie es Botero ausgedrückt hatte ${ }^{56}$ - um die „,conservatio status" oder die ,stabilitas imperii" sprechenden Zieltopoi aus der nunmehr wieder lateinisch geschriebenen Politikliteratur Deutschlands lauteten. Stabilität und Dauerhaftigkeit der Herrschaft wurden im 17. Jahrhundert zum zentralen Leitmotiv der Politiktheorie, an dem das Arsenal der von den Politici vorgeschlagenen Handlungsstrategien ausgerichtet wird. ${ }^{57}$ Der Zieltopos von der conservatio status tendiere, so hat es Dreitzel jüngst auf den Nenner gebracht, „zur Isolierung des Problems der Machterhaltung des Herrschers von allen übrigen politischen Aufgaben". ${ }^{58}$

Freilich muß hier immer bewußt bleiben, daß diese neuartige Machtlehre in Deutschland nur teilweise und vielfach auch nur unter Angleichungen an das ältere Politikkonzept rezipiert wurde. Die gemeinhin in der ,politica christiana" zusammengefaßten Politikautoren wie etwa Dietrich Reinkingk, aber auch noch Veit Ludwig von Seckendorff, lehnten sie sogar insgesamt ab und beharrten auf der Weiterführung der überlieferten Politikleitlinien. Aber dies kann hier beiseite gelassen werden, denn in diese traditionellen Politikkonzepte fanden die innovativen Elemente der politischen Theorie im 17. Jahrhundert kaum Eingang. Das beunruhigende und herausfordernde Neue, auf das die Politiklehre im 17. Jahrhundert mit einer wahren Flut von Publikationen reagierte, ${ }^{59}$ lag vielmehr in der neuen, machtorientierten Lehre von der Ratio status. Eine ganz wesentliche Provokation dieses Politikverständnisses bestand nun gerade darin, daß es in Gegensatz zu dem überlieferten Gemeinwohlgedanken geraten mußte. Denn die traditionelle Gemeinwohltopik dachte und argumentierte im Interesse des Gemeinwesens als

\footnotetext{
53 Simon 2002, Teil C, Kap. II.1.

54 Botero 1930, Buch I, Kap. I.

$55 \mathrm{Vgl}$. Stolleis 1983 b, S. $70 \mathrm{ff}$.

${ }^{56}$ Botero 1930, Buch I, Kap. I.

57 Vgl. Weber 1992, S. 321 und S. 105; Behnen 1984, S. 427 und 435; Dreitzel 1995, S. 139; Dreitzel 1991, S. 572; Seils 1968, S. 136.

${ }^{58}$ Dreitzel 1995, S. 140.

59 Stolleis 1988, S. 110.
} 
ganzem; die Bezugsgröße war die soziale Gemeinschaft insgesamt und gerade nicht der Machtstatus des Fürsten.

Betrachtet man nun, wie die Gemeinwohlformeln in einem derartigen Kontext eingesetzt werden, so zeigt sich folgendes: Diejenigen Autoren, die die neue, machtorientierte Politik im wesentlichen übernehmen, führen die Gemeinwohltopoi so in ihre Texte ein, daß sie die neuen Machtstrategien legitimieren. Die Argumentation ist dabei typischerweise auf den Gesichtspunkt der Friedenssicherung konzentriert, wobei das Wort „Friede" nunmehr bei seiner Verwendung im Kontext der hier in Rede stehenden Politikliteratur einen seinem modernen Verständnis entsprechenden Gehalt angenommen hat: „Friede“ ist ein Zustand innerer Sicherheit und Ruhe, bedeutet also im wesentlichen nur die absentia belli; der Bezug zur mittelalterlichen Ordnungsvorstellung der tranquillitas ordinis ist gekappt. ${ }^{60}$ Die Friedenssicherung im Sinne einer vom Fürst zu erzwingenden securitas publica ist in dieser Form der Politiklehre der entscheidende legitimatorische Gesichtspunkt, mit dem die Notwendigkeit starker, handlungsfähiger Herrschaft begründet und gerechtfertigt wird; das Gemeinwohlargument tritt hierbei in engen Bezug zu diesem Gesichtspunkt: Das Gemeinwesen sei, so das verbreitete Argumentationsmuster, wie man es auch bei Justus Lipsius finden kann, in so grundlegender Weise von innerer und äußerer Gewalt bedroht, daß eine starke, machtvolle und daher durchsetzungsfähige Herrschaft zwingend notwendig sei - eine Herrschaft, die auf Grund ihrer Fähigkeit zur effektiven Friedenssicherung das staatliche Gewaltmonopol $\mathrm{zu}$ erzwingen und auf diese Weise das Überleben jedes einzelnen Individuums in der Gemeinschaft zu sichern vermag. Lipsius betont in fast beschwörenden Worten die ihm zufolge unabweisbare Notwendigkeit einer starken Herrschaftsgewalt - notwendig, weil andernfalls das Gemeinwesen von der inneren Gewalt auseinandergesprengt und zersetzt würde: „sine imperio enim nec domus ulla, nec civitas, nec gens, nec hominum universum genus stare potest " ${ }^{61}$ Jede societas, sei es die häusliche, sei es die politische, zerfällt oder wird Beute äußerer Feinde ohne das „vinculum“ starker Herrschaft, ohne die „potentia“ oder die „dominatio“ des Fürsten, die sie zusammenhält. Hier liegt der Punkt, an dem diese Strömung politischen Denkens den Gemeinwohltopos einsetzt: Die Macht des Fürsten, seine finanzielle und militärische Stärke, dient insofern auch dem ganzen Gemeinwesen, als nur sie Anarchie und Bürgerkrieg zu unterdrücken vermag. Große Teile der Politiklehre des 17. Jahrhunderts sind getragen von einer gravierenden Einschätzung der Gefahr innerer Gewalt, die als ständig drohender politischer Katastrophenfall die Aufrüstung der Herrschaft rechtfertigt. Das ,,augere potentiam“, das diese Politiklehre propagiert und zu dem sie die Handreichungen geben möchte, ist auch im Interesse des Gemeinwohls, weil nur die Macht und Durchsetzungskraft des Fürsten das fundamentale Gemeinschaftsgut des inneren Friedens sichern kann. ${ }^{62}$ Der Aspekt der Herrschaftsbehauptung wird hier so dominant, daß der Leitgesichtspunkt des bonum commune weitgehend darin aufgeht. ${ }^{63}$ Das Gemeinwohl ist in diesem Kontext zentriert auf das Erfordernis starker, durchsetzungsfähiger Herrschaft.

\footnotetext{
${ }^{60}$ Vgl. Conze 1984, S. 839; Meyers 1992, S. 89; Janssen 1975, S. 557.

${ }^{61}$ Lipsius 1589, Buch II, Kap. 1; weitere Nachweise bei Simon 2002, Teil C, Kap. II.4.

62 Weber 1992, S. 331 ff.

${ }^{63}$ Dreitzel 1970, S. 124.
} 
In einem derartigen Argumentationsmuster erscheint aber nicht nur die Stärkung der herrschaftlichen Machtpotenzen als gemeinwohlbezogenes politisches Ziel angeraten, sondern ganz generell die Ausweitung der herrschaftlichen Handlungsmöglichkeiten, um deren Effektivität im Interesse aller zu steigern. Auch dies geschieht unter intensivem Rückgriff auf die Gemeinwohlformeln: In bestimmten politischen Ausnahmesituationen muß sich der Herrscher im Interesse des Gemeinwohls über die normativen Vorgaben von Recht, Moral und Religion hinwegsetzen können. ${ }^{64}$ Individuelle Rechte muß er verletzen, Betrug, List und Täuschung im politischen Handeln praktizieren können, wenn dies im Ausnahmefall eines drohenden Zusammenbruchs der inneren Ordnung und des Friedens notwendig wird: „Multa enim vel ex necessitate vel boni publici causa permittuntur, quae alia non probarentur." Diese Form des Gemeinwohlrekurses, hier zitiert aus der Doctrina Politica (Buch II, Kap. 5) des Henning Arnisäus, findet sich in entsprechenden Wendungen in vielen Politica des 17. Jahrhunderts. Zugleich wird hier sichtbar, wie dicht sich das bonum publicum in diesem Kontext der necessitas annähert. ${ }^{65}$ Gemeinwohl und necessitas gehen hier in ihrem Gehalt nahezu ineinander auf. Der Topos der necessitas nahm in der machtorientierten Politiklehre des 17. Jahrhunderts einen zentralen Stellenwert ein; ${ }^{66}$ damals entstand eine ausgefeilte Topik der $n e-$ cessitas, der es vor allem um die Erweiterung der politischen Handlungsspielräume gegenüber den rechtlichen Begrenzungen zu tun ist: ${ }^{67}$ Die Infragestellung des inneren Friedens, die Situation drohenden Bürgerkriegs, verlangt danach, die Politik aus den Fesseln des Rechts zu lösen, denn im Moment außerordentlicher Gefahr muß die Politik so beweglich sein, daß sie auch nach den Kriterien der Effizienz, nicht nur nach denjenigen des Rechts zu handeln imstande ist. ${ }^{68}$

Die Gemeinwohlargumentation dieser Form der Politikliteratur rückt also die herrschaftslegitimierende Funktion in den Vordergrund. Ihre zentrale Bedeutung lag hier weniger in der normativen Festlegung des Herrschers, sondern in der Erweiterung des politischen Handlungsspielraums durch die Legitimierung von Rechtsdurchbrechungen, Listen und Arkanpolitik. Die Politiklehre dieser Zeit empfiehlt dem Fürsten deshalb auch - so kann man es etwa in der Arkan-Politik Arnold Clapmars nachlesen - das Gemeinwohlargument bewußt zu instrumentalisieren und ganz gezielt zur Legitimierung von Rechtsverletzungen einzusetzen, um dadurch die Akzeptanz solcher Maßnahmen zu sichern. ${ }^{69}$

Die Akzentuierung des Gemeinwohlargumentes ändert sich freilich, sobald man statt der machttheoretisch orientierten Politikliteratur solche Autoren in den Blick nimmt, die zumindest partiell auf der Sichtweise und den Wertungen der traditionellen Politik beharren, das Neue aber auch nicht - wie etwa die Vertreter der politica christiana rundweg ablehnen, sondern eine Verbindung beider Formen politischen Denkens suchen. Namentlich wären hier vor allem Hermann Conring und - zeitlich früher - Hen-

${ }^{64}$ Hierzu eingehend Behnen 1987, S. 147f. und S. 166.

65 Stolleis 1983 b, S. 93.

${ }^{66}$ Hierzu Boldt 1972, S. 346ff.; Kluxen 1967.

67 Pichler 1983, S. 47f.; Münkler 1987, S. 190 f.

68 Dreitzel 1991, S. 576.

${ }^{69}$ Behnen 1987, S. 166, Fn. 146. 
ning Arnisäus zu nennen. Bei diesen Autoren wird das Gemeinwohlargument dazu verwendet, die neue Politiklehre dem älteren politischen Denken anzugleichen. Das geschieht hier dadurch, daß den neuen, aus dem Kontext machtpolitischen Denkens stammenden politischen Leitbegriffen wie der Ratio Status durch ihre Verbindung mit dem traditionsgesättigten Gemeinwohltopos die Brisanz genommen wird. Auf diese Weise kam man dann zu einer Unterscheidung von guter und schlechter Staatsräson: Die „gute Staatsräson“ zeichnet sich - so findet man es etwa bei Besold - dadurch aus, daß sie auf das bonum publicum achtet, während die „schlechte Staatsräson“ nur das bonum privatum des Herrschers im Auge hat. ${ }^{70}$ Hier tritt wieder der normativ bindende Gehalt des Gemeinwohlargumentes hervor: Der Herrscher, der nur seine persönliche Macht im Auge hat, handelt nicht im Interesse des Gemeinwesens. Auf diese Weise wird die machtorientierte Status-Politik mit dem herkömmlichen politischen Denken harmonisiert. Zugleich wird der vom Standpunkt des konventionellen Politikverständnisses aus anrüchige Begriff der Ratio Status an das herkömmliche Politikmuster zurückgebunden.

\section{Die Verwendung des Gemeinwohltopos als Ausdruck von Herrschaftsintensivierung}

Der gemeinsame Nenner der verschiedenartigen Anwendungsfelder des Gemeinwohlargumentes liegt in der Intention, intensivierte und funktional erweiterte Herrschaft einerseits normativ anzuleiten, andererseits zu legitimieren und konsensfähig zu machen. Die Intensivierung der Herrschaft ergab sich dabei zunächst aus der neuartigen Funktion legislativer Steuerung des Gemeinwesens, die seit dem Hochmittelalter in den Kommunen und dann auch in den sich allmählich formierenden Territorien als neues staatliches Aufgabenfeld erscheint. Im Zuge der politiktheoretischen Umorientierung, wie sie im Aufkommen der Staatsräsonlehre zu Beginn der Neuzeit sichtbar wird, rückt der Gesichtspunkt einer möglichst starken, durchsetzungsfähigen und normativ ungebundenen Herrschaft in den Vordergrund. Zur Legitimierung der hierfür erforderlichen Durchbrechung moralisch-religiöser und rechtlicher Normen wird wiederum in verstärktem Maße auf den Gemeinwohltopos zurückgegriffen; der Aspekt normativer Festlegung politischen Handelns tritt dabei zurück. In beiden Fällen kommt es jedenfalls zu einer Relativierung des überlieferten Rechts, das sowohl durch Gesetzgebung als auch durch necessitär, mit der Sicherung der politischen Handlungsfähigkeit begründete Durchbrechungen zur Disposition gestellt wird. In beiden Fällen wird auch der politische Handlungsspielraum gegenüber dem Recht erweitert, das seinerseits zum Produkt zielgerichteten politischen Handelns wird. Die solchermaßen bedingte Herrschaftsintensivierung erhöht aber auch die Anforderungen an die Integrationsfähigkeit der sozialen Gemeinschaften; den verstärkten Rückgriff auf den Gemeinwohltopos, wie er in diesem Kontext zu beobachten ist, kann man als Ausdruck eines erhöhten Integrationsdrucks deuten. Er wird notwendig, weil die Eingliederung und Unterordnung der Individuen in

${ }^{70}$ Besold 1641, S. 12; Stolleis 1983a, S. 79. 
eine mit erweiterten Regelungs- und Steuerungskompetenzen ausgestattete soziale Einheit schwieriger zu bewerkstelligen ist, als dies bei einer nur locker integrierten politischen Gemeinschaft der Fall wäre. Eine solche erhöhte Integrationsleistung kann nur erbracht werden, wenn mit der Intensivierung der Herrschaft auch deren Akzeptanz gesteigert werden kann. Hierin dürfte die entscheidende Bedeutung des Gemeinwohltopos liegen: Seine zunehmende Verbreitung in der politischen und rechtlichen Sprache seit dem Hochmittelalter indiziert ein verstärktes Bemühen um Akzeptanz sich intensivierender Herrschaft und das Bestreben, deren Integrationsfähigkeit zu steigern.

\section{Quellen}

Aegidius Romanus (1556), De regimine principum libri III, Ausgabe Rom (ND Frankfurt/M. 1968).

Arnisäus, H. (1605), Doctrina Politica in genuinam Methodum quae est Aristotelis reducta, Frankfurt/M.

Augustin (1981), De civitate Dei, hg. von Bernhard Dambach und Alfons Kalb, 2 Bde. Darmstadt.

Besold, Chr. (1641), De Arcanis rerumpublicarum dissertatio, Amsterdam.

Botero, G. (1930), Della Ragion di Stato. Delle cause della grandezza città, hg. von C. Morandi, Bologna.

Ferrarius, Joh. (1533), Von dem Gemeinen Nutze, Ausgabe Marburg.

Lipsius, J. (1589), Libri sex politicarum sive Doctrina civilis. Editio in Cura Matthiae Berneggeri, Frankfurt/M. und Leipzig 1704.

Tolomäus von Lucca (1948), De regimine principum (Fortsetzung des Fürstenspiegels von Thomas von Aquin, De regimine principum), hg. von Joseph Mathis, Turin.

\section{Literatur}

Anton, H. H. (1989), Stw. „Fürstenspiegel“, Lateinisches Mittelalter, in: Lexikon des Mittelalters, Bd. 4, Sp. 1040-1048.

Bader, K. S. (1962), Dorfgenossenschaft und Dorfgemeinde (Studien zur Rechtsgeschichte des mittelalterlichen Dorfes, Bd. 2), Weimar.

Behnen, M. (1984), Herrscherbild und Herrschaftstechnik in der „Politica“ des Johannes Althusius, in: Zeitschrift für Historische Forschung 11, S. 417-472.

Behnen, M. (1987), Arcana - haec sunt Ratio Status, in: Zeitschrift für Historische Forschung 14, S. 129-195.

Berges, W. (1938), Die Fürstenspiegel des hohen und späten Mittelalters, Stuttgart.

Blickle, P. (1986), Kommunalismus, Parlamentarismus, Republikanismus, in: Historische Zeitschrift 242, S. 529-556.

Boldt, H. (1972), Stw. „Ausnahmezustand“", in: Geschichtliche Grundbegriffe. Historisches Lexikon zur politisch-sozialen Sprache in Deutschland, Bd. 1, hg. von Otto Brunner/ u. a., Stuttgart, S. 343-376.

Conze, W. (1984), Stw. „Sicherheit“, in: Geschichtliche Grundbegriffe. Historisches Lexikon zur politisch-sozialen Sprache in Deutschland, Bd. 5, hg. v. O. Brunner u. a., Stuttgart, S. 831-862.

Dreitzel, H. (1970), Protestantischer Aristotelismus und absoluter Staat. Die „Politica“ des Henning Arnisaeus (ca. 1575-1636) (=Veröffentlichungen des Instituts für Europäische Geschichte, Mainz 1955), Wiesbaden. 
Dreitzel, H. (1991), Monarchiebegriff in der Fürstengesellschaft. Semantik und Theorie der Einherrschaft in Deutschland von der Reformation bis zum Vormärz, Köln/Böhlau.

Dreitzel, H. (1995), Die „Staatsraison“ und die Krise des politischen Aristotelismus. Zur Entwicklung der politischen Philosophie in Deutschland im 17. Jahrhundert, in: Aristotelismo politico e ragion di stato. Atti del convegno internazionale di Torino 11-13 febbraio 1993, hg. von A. E. Baldini, Florenz, S. 129-156.

Eberhard, W. (1985), „Gemeiner Nutzen“ als oppositionelle Leitvorstellung im Spätmittelalter, in: Renovatio et Reformatio. FS für Ludwig Hödl zum 60. Geburtstag, hg. von M. Gerwig und G. Ruppert, Münster, S. 195-214.

Eberhard, W. (1986), Der Legitimationsbegriff des „Gemeinen Nutzens" im Streit zwischen Herrschaft und Genossenschaft im Spätmittelalter, in: Zusammenhänge, Einflüsse, Wirkungen. Kongressakten zum ersten Symposium des Mediävistenverbandes in Tübingen, hg. von J. O. Fichte, K. H. Göller, B. Schimmelpfennig, S. 241-254, Berlin.

Eberhard, W. (1988), Kommunalismus und Gemeinnutz im 13. Jahrhundert, in: Gesellschaftsgeschichte. FS für Karl Bosl zum 80. Geburtstag, hg. i. A. des Collegium Carolinum von F. Seibt, Bd. 1, München, S. 271-294.

Fried, P. (1969), Zur „staatsbildenden“ Funktion der Landfrieden im früheren bayerischen Territorialstaat, in: Festschrift für Max Spindler zum 75. Geburtstag, hg. von D. Albrecht, A. Kraus, K. Reindl, München, S. 283-306.

Gagnér, St. (1960), Studien zur Ideengeschichte der Gesetzgebung, Uppsala.

Gierke, O. v. (1868), Das deutsche Genossenschaftsrecht, Bd. 1: Rechtsgeschichte der deutschen Genossenschaft, ND Graz 1954.

Hibst, P. (1991), Utilitas Publica - Gemeiner Nutz - Gemeinwohl. Untersuchungen zur Idee eines politischen Leitbegriffes von der Antike bis zum späten Mittelalter, Frankfurt/M.

Jakobs, H. (1994), Kirchenreform und Hochmittelalter 1046-1215 (Oldenbourg Grundriss der Geschichte Bd. 7), 3. Aufl. München.

Janssen, W. (1975), Stw. „Friede“, in: Geschichtliche Grundbegriffe. Historisches Lexikon zur politisch-sozialen Sprache in Deutschland, Bd.2, Stuttgart, S. 543-591.

Kaufmann, E. (1979), Stw. „Landfriede I (Landfriedensgesetzgebung)“, in: Handwörterbuch zur deutschen Rechtsgeschichte, hg, von A. Erler und E. Kaufmann, Bd. 2, Berlin, Sp. 1451-1465.

Kluxen, K. (1967), Politik und Existenz bei Machiavelli, dargestellt am Begriff der Necessità, Stuttgart.

Le Goff, J. (1965), Das Hochmittelalter (Fischer Weltgeschichte Bd. 11), Frankfurt/M.

Maier, H. (1980), Die ältere deutsche Staats- und Verwaltungslehre, 2. Auflage, München.

Meyers, R. (1992), Die Signatur der Neuzeit - Machiavelli, Hobbes und die legitimatorische Begründung des modernen Staates als Ordnungsmacht, in: Politische Philosophie und Erkenntnistheorie (Studien zur Politikwissenschaft IV), hg. von N. Konegen, Münster, S. 77-118.

Miethke, J. (1991), Politische Theorien im Mittelalter, in: Politische Theorien von der Antike bis zur Gegenwart, hg. von H. J. Lieber, München, S. 47-156.

Miethke, J. (1992), Politische Theorie in der Krise der Zeit. Aspekte der Aristotelesrezeption im früheren 14. Jahrhundert, in: Institutionen und Geschichte. Theoretische Aspekte und mittelalterliche Befunde (Norm und Struktur. Studien zum sozialen Wandel in Mittelalter und früher Neuzeit Bd. 1), hg. von G. Melville, Köln u. a., S. 157-186.

Mohnhaupt, H. (1972), Potestas legislatoria und Gesetzesbegriff im Ancien Régime, in: Ius Commune 4, S. $188-239$. 
Münkler, H. (1987), Im Namen des Staates. Die Begründung der Staatsraison in der Frühen Neuzeit, Frankfurt/M.

Pichler, Joh. W. (1983), Necessitas. Ein Element des mittelalterlichen und neuzeitlichen Rechts, Berlin.

Schneider, J. H. J. (1990), Thomas von Aquin und die Grundlegung der politische Philosophie in „De Regno", in: Rechts- und Sozialphilosophie des Mittelalters (Salzburger Schriften für Rechts-, Staats- und Sozialphilosophie, Bd. 12), hg. von E. Mock u. G. Wieland, Frankfurt/M., S. 47-63.

Scholz, R. (1903), Die Publizistik zur Zeit Philipps des Schönen und Bonifaz' VIII (Kirchenrechtliche Abhandlungen 6/8), Stuttgart.

Schulze, W. (1986), Vom Gemeinnutz zum Eigennutz. Über den Normenwandel in der ständischen Gesellschaft der frühen Neuzeit, in: Historische Zeitschrift 243, S. 598-625.

Seils, E.-A. (1968), Die Staatslehre des Jesuiten Adam Contzen, Beichtvater Kurfürst Maximilians I. von Bayern (Historische Studien 405), Lübeck/Hamburg.

Sellin, V. (1978), Stw. „Politik“, in: Geschichtliche Grundbegriffe. Historisches Lexikon zur politischsozialen Sprache in Deutschland, Bd. 4, Stuttgart, S. 789-874.

Simon, Th. (1997), Krise oder Wachstum? Erklärungsversuche zum Aufkommen territorialer Gesetzgebung am Ausgang des Mittelalters, in: Wirkungen europäischer Rechtskultur. Festschrift für Karl Kroeschell zum 70. Geburtstag, hg. von G. Köbler u. H. Nehlsen, München, S. 1201-1217.

Simon, Th. (2002), Policey im kameralistischen Verwaltungsstaat: das Beispiel Preußen, in: Policey und frühneuzeitliche Gesellschaft, Frankfurt/M. hg. von K. Härter, S. 473-496.

Stolleis, M. (1980), Arcana Imperii und Ratio Status. Bemerkungen zur politischen Theorie des frühen 17. Jahrhunderts, in: Ders., Staat und Staatsräson in der frühen Neuzeit: Studien zur Geschichte des öffentlichen Rechts, Frankfurt/M. 1990, S. 37-72.

Stolleis, M. (1981), Friedrich Meineckes „Die Idee der Staatsräson“ und die neuere Forschung, in: Ders., Staat und Staatsräson in der frühen Neuzeit: Studien zur Geschichte des öffentlichen Rechts, Frankfurt/M. 1990, S. 134-164.

Stolleis, M. (1983a), Machiavellismus und Staatsräson. Ein Beitrag zu Conrings politischem Denken, in: Ders., Staat und Staatsräson in der frühen Neuzeit: Studien zur Geschichte des öffentlichen Rechts, Frankfurt/M. 1990, S. 73-105.

Stolleis, M. (1983b), Pecunia nervus rerum. Zur Staatsfinanzierung in der frühen Neuzeit, Frankfurt/M.

Stolleis, M. (1987), Lipsius-Rezeption in der politisch-juristischen Literatur des 17. Jahrhunderts in Deutschland, in: Ders., Staat und Staatsräson in der frühen Neuzeit: Studien zur Geschichte des öffentlichen Rechts, Frankfurt/M. 1990, S. 232-267.

Stolleis, M. (1988), Geschichte des öffentlichen Rechts in Deutschland, Bd. 1: Reichspublizistik und Policeywissenschaft 1600-1800, München.

Struve, T. (1978), Die Entwicklung der organologischen Staatsauffassung im Mittelalter (Monographien zur Geschichte des Mittelalters 16), Stuttgart.

Viroli, M. (1992), From politics to reason of State. The acquisition and transformation of the language of politics 1250-1600, Cambridge.

Vogel, O. (1953), Der ländliche Einung nach den zürcherischen Rechtsquellen, Aarau.

Weber, W. (1992), Prudentia gubernatoria. Studien zur Herrschaftslehre in der deutschen politischen Wissenschaft des 17. Jahrhunderts, Tübingen. 